\title{
Effective Intervention in Reducing Depression in General Population during Pandemic of Covid-19
}

\author{
M. Askar ${ }^{1 *}$ Ernawati $^{2}$ Anshar $^{2}$ \\ ${ }^{1,3}$ Health Polytechnic of Ministry of Health in Makassar, Indonesia \\ ${ }^{2}$ Nani Hasanuddin Makassar College, Indonesia \\ *askar@poltekkes-mks.ac.id
}

\begin{abstract}
The purpose of this narrative review is to provide an important overview of effective interventions in reducing depression to make it easier for doctors, nurses, and other health professionals to plan appropriate interventions to overcome and reduce depression in the general population based on evidence-based practice. The research method was carried out by conducting a quality research exploration on the research database via pubmed.gov. The types of articles selected were limited to articles published during 2020 and those related to depression due to the Covid-19 pandemic. The results showed that out of 410 articles obtained using the keyword "effective intervention for depression during pandemic covid-19", there were 3 (three) articles that relevant to the purpose of this review. Conclusion. Regular exercise activities and togetherness with family in carrying out daily activities accompanied by clean and healthy living behaviors can reduce depression and maintain a lower level of depression during the Pandemic of Covid-19.
\end{abstract}

Keywords:Depression; Effective intervention; Covid-19

\section{Introduction}

The Covid-19 pandemic that has occurred since December 2019 in Wuhan China has had a huge impact on human life globally, not only causing physical health problems but also triggering psychological disorders, one of which is depression. Covid-19 in the general population causes depression commonly at a moderate level. ${ }^{1}$ Many studies have been conducted to look at the prevalence of psychological disorders during the Covid-19 Pandemic in 2020 and generally show a significant increase compared to before the Covid19 Pandemic. $^{2}$

The incidence of depression during the Covid-19 pandemic in the general population has increased significantly. Depression in the general population in China increases significantly with age, chronic illnesses, low income, the effects of the pandemic, and worries about suffering from Covid 19 during the Covid-19 pandemic. $^{3}$ The results of a systematic review on 14 studies with a sample size of 44,531 people showed that the prevalence of depression was $33.7 \%{ }^{4}$, other studies varied between $14,6 \%-48,3 \% .^{2}$ An increase in psychological disorders including depression, stress and anxiety have been shown to occur in the
UK especially in younger people, women, and individuals identified as a group at risk for Covid19 exceeding the incidence in the normal population. ${ }^{5}$

Depression was found to be higher in women aged 18-19 years, single, students, and had a low income compared to the expenditure among others of the general population. This is related to their habit of using social media compared to others, getting excessive and inaccurate information that can trigger depression. Those who moved during the quarantine period, experienced loneliness, fear of dying, helplessness, sleep disturbances, felt useless and worthless, started smoking and drinking alcohol, experienced moderate depression. $^{1}$

Depression is predicted to result in an increase in alcohol consumption and Cannabis use in adolescents. Handling the harmful effects of Covid-19 on mental health must be a priority for international public health. Interventions that are effective in reducing the occurrence of depression in people affected by the Covid-19 pandemic need to be explored and summarized from various quality studies. 


\section{Methods}

From the exploration results via pubmed.gov by entering the keyword "effective intervention for depression during the Covid-19 pandemic", 401 articles were obtained. Of the 401 articles, they were reviewed one by one to find research articles that presented effective interventions in reducing depression during the Covid-19 pandemic and obtained 3 relevant articles. The selection of articles was carried out following the research objectives (purposive sampling). This research was conducted in December 2020.

\section{Results}

From the results of a review of 401 articles, 3 articles were obtained related to effective interventions in reducing depression that occurred in people affected by the Covid-19 pandemic. The following articles are each as follows:

1. "Beneficial Effects of Exercise on Depression and Anxiety During the Covid-19 Pandemic: A Narrative Review". From this article, it was found that increasing light activity and reducing behavior can be beneficial in reducing depressive behavior. Regular exercise also has benefits against depression. Physical exercise outside the home if not available during the Covid-19 Pandemic, can be done by exercising indoors. This is recommended because it can relieve depression and anxiety, in addition to improving the immune system. Exercise strategies can be done with a duration of 150 minutes of moderate-intensity activity or 75 minutes of vigorous-intensity per week or do both with modifications according to each individual's ability. Home exercises that can be done include squats, knees to elbows, side knee lifts, planks, back extensions, "superman," chair dips, "Bridge," seated meditation, chest opener, and legs up the wall. Activities outside the home can be carried out according to local government policies in the field or park while maintaining a physical distancing. Various exercise programs can be carried out, including aerobic exercise, balance training, resistance, coordination, and other exercise activities. When carrying out exercises outside the home, it is recommended not to use public available training equipment to prevent transmission of the virus. Thus regular physical activity during a pandemic is very beneficial in dealing with depression. ${ }^{6}$

2. "Determining depression and related factors in a society affected by COVID-19 pandemic". This article is not an intervention research study, but a descriptive study with a crosssectional design. However, something is interesting from this study where it was found that the depression score of those who spent time with their family, spent time with them, were busy with studying at home, or worked had lower depression than the others. Besides, those who carried out the behavior of walking, exercising, sleeping regularly, and consuming a balanced diet, and praying had lower levels of depression than others. Thus from this study, it is concluded that depression can be reduced by carrying out joint activities with family and daily activities carried out from home and that clean and healthy living habits are beneficial in reducing and maintaining low levels of depression during the Covid-19 Pandemic. ${ }^{1}$

3. "A longitudinal study on the mental health of the general population during the COVID-19 epidemic in China". This study is a longitudinal study and not research related to the effectiveness of the intervention. However, the results of this study can provide an overview of the basis for effective interventions in reducing the occurrence of depression that occurs in the general population. The results of this study indicate that there are no significant changes in the levels of stress, anxiety, and depression between the start of the pandemic and the four weeks thereafter. However, it is interesting that there are protective factors that include high trust in doctors, perception of the possibility of survival, low risk of suffering from Covid-19, satisfaction with health 
information, and personal precautions. ${ }^{7}$ This study suggests interventions that are based on research results that to reduce depression in the general population, they need to be provided with unbiased knowledge about Covid-19, teach correct coping methods, ensure the availability of health services and daily necessities and financial support during the Covid-19 Pandemic.

\section{Discussions}

This research has explored various important and quality articles related to interventions in reducing depression that occur in society as presented in the description of the research results. Literature 1 shows that an effective intervention in reducing and overcoming depression during the Covid-19 pandemic is to carry out regular exercise activities. Exercise activities can be done through activities with moderate intensity or with strong intensity. Activities can be carried out at home or outside the home with due observance of government policies regarding restrictions on activities outside the home. This has proven to be effective in reducing and overcoming depression due to the Covid-19 pandemic.

However, several interventions, especially from literature 2 and 3, are still the thoughts of the analysis of the literature and have not been tested for their effectiveness. Both kinds of literature were included because there was still a lack of literature that could prove the effectiveness of interventions in reducing depression during the Covid-19 Pandemic in the community. In literature 2, the role of the family as a support system is very important in reducing and overcoming depression during the Covid-19 pandemic. Before the Covid-19 pandemic, most people were busy with activities outside the home and many of them only spent a little time with their nuclear family at home. The occurrence of the Covid-19 pandemic which was followed by a lockdown policy or restrictions on activities outside the home created a new situation where families became more often together at home. This togetherness should be optimized by doing activities together and creating intensive and happy interactions to reduce depression due to various risk factors.

From the third literature, it seems that health information is important for depression during the Covid-19 pandemic. Excessive information exposure makes adolescents the largest group of social media users, making this group experience the highest depression compared to other groups. However, it appears that this can be different when the information obtained is satisfied, unbiased and not exaggerated. The quality of health information is of key importance in maintaining the mental health of the general population and reducing depression. The information available in the mass media needs to endeavor in such a way as to reduce information that is a hoax in nature and misleading, unbiased, and the quality of information should be able to satisfy the needs of the general population.

Important psychological support is given to patients suffering from Covid-19 and health workers who work with Covid 19 patients, however, the mental health of the general population also requires important attention. The research articles obtained in general are more focused on the prevalence of psychological disorders due to the Covid-19 pandemic and not much research has been done to find solutions to reduce depression that occurs in the general population due to the Covid-19 pandemic.

\section{Conclusion}

Regular exercise activities and togetherness with family in carrying out daily activities accompanied by clean and healthy living behaviors can reduce depression and maintain a lower level of depression during the Covid-19 pandemic.

\section{Limitations and Future Studies}

Researchers need to study more to find solutions to depressive conditions occurring in the general population rather than obtaining data only regarding the prevalence of health mental disturbances. 


\section{References}

[1] Ustun G. Determining depression and related factors in a society affected by COVID-19 pandemic. Int J Soc Psychiatry. 2020 Jun 30:20764020938807. doi: 10.1177/0020764020938807. Epub ahead of print. PMID: 32605422; PMCID: PMC7331110.

[2] Vindegaard N, Benros ME. COVID-19 pandemic and mental health consequences: Systematic review of the current evidence. Brain Behav Immun. 2020 Oct;89:531542. doi: 10.1016/j.bbi.2020.05.048. Epub 2020 May 30. PMID: 32485289; PMCID: PMC7260522.

[3] Ping W, Zheng J, Niu X, Guo C, Zhang J, Yang H, Shi Y. Evaluation of healthrelated quality of life using EQ-5D in China during the COVID-19 pandemic. PLoSOne. 2020 Jun 18;15(6):e0234850. doi: $\quad$ 10.1371/journal.pone.0234850. PMID: 32555642; PMCID: PMC7302485.

[4] Salari N, Hosseinian-Far A, Jalali R, Vaisi-Raygani A, Rasoulpoor S, Mohammadi M, Rasoulpoor S, KhalediPaveh B. Prevalence of stress, anxiety, depression among the general population during the COVID-19 pandemic: a systematic review and meta-analysis. Global Health. 2020 Jul 6;16(1):57. doi:10.1186/s12992-020-00589-w. PMID: 32631403 ; PMCID: PMC7338126.

[5] Jia R, Ayling K, Chalder T, Massey A, Broadbent E, Coupland C, Vedhara K. Mental health in the UK during the COVID-19 pandemic: cross-sectional analyses from a community cohort study. BMJ Open. 2020 Sep 15;10(9):e040620. doi: $\quad 10.1136 /$ bmjopen-2020-040620. PMID: 32933965; PMCID: PMC7493070.

[6] Hu S, Tucker L, Wu C, Yang L. Beneficial Effects of Exercise on Depression and Anxiety During the Covid-19 Pandemic: A Narrative Review. Front Psychiatry. 2020 Nov 4;11:587557. doi: 10.3389/fpsyt.2020.587557. PMID: 33329133; PMCID: PMC7671962.

[7] Wang C, Pan R, Wan X, Tan Y, Xu L, McIntyre RS, Choo FN, Tran B, Ho R, Sharma VK, Ho C. A longitudinal study on the mental health of general population during the COVID-19 epidemic in China. Brain Behav Immun. $2020 \mathrm{Jul} ; 87: 40-48$. doi: 10.1016/j.bbi.2020.04.028. Epub 2020 Apr 13. PMID: 32298802; PMCID: PMC7153528. 